\title{
Extractions of size granding classes in undersize
}

\author{
Oleksandr Poluliakh ${ }^{1,}$, Danylo Poluliakh ${ }^{2}$, Serhii Kostyria ${ }^{3}$, and Liudmyla Kabakova ${ }^{3}$ \\ ${ }^{1} \mathrm{ME}$ «Ukrndivuhlezbahachennya» SE «Vuhleinnovatsiya», 49000, Dnipro, Volodymyra Monomakha \\ Str., 12/413, Ukraine \\ ${ }^{2}$ National Technical University "Dnipro Polytechnic”, 49005, Dnipro, Dmytra Yavornytskoho Ave., \\ 19, Ukraine \\ ${ }^{3}$ Institute of Geotechnical Mechanics named by N. Poljakov of National Academy of Sciences of \\ Ukraine, 49005, Dnipro, Simferopolska Str., 2a, Ukraine
}

\begin{abstract}
On the basis of averaging of 544 balances of grain-size screening products distribution of the largeness classes extraction coefficients are set in a subsize product at dry and wet preparatory screening of ordinary coal and shallow machine class deslurring. Findings can be used for determination of indexes of the indicated technological operations at the calculations of quality-quantitative and water-slime coal preparations factories charts.
\end{abstract}

\section{Introduction}

Process parameters of coal preparation plant operation depend to a large extent upon efficiency of preparation of run-of-mine coal machine grades prescribing its distribution through the preparation processes. This distribution shall be taken into account, when the actual balance of coal preparation products and qualitative and quantitative, as well as water-sludge flow charts of the designed or modified coal preparation plants are computed.

Actual data of plant operation with the equivalent raw material and equipment, coal preparation plant design codes [1], as well as different regulatory documents $[2,3]$ and scientific literature [4-6] are used for their computation. However, variation of coal quality towards increase of rock content, moisture content and fine grade content has caused obsolescence of previously used rates. Computation of consistency of size grade distribution through products separation in process of preparation of run-of-mine coal machine grades under the existing conditions shall be the actual research and production task and its addressing shall facilitate approximation of design and actual coal preparation parameters.

Machine grade preparation at the coal preparation plants shall generally include tree process operations: dry screen splitting, large-size grain machine grading, fine grain machine grading.

The first process operation is performed with dry screening, the second - with wet screening, the third - with deslurring.

\footnotetext{
${ }^{*}$ Corresponding author: tehotdel.ukrnii@gmail.com
} 


\section{Methods}

To compute parameters of the above process operations, it shall be required to evaluate size grades of the feedstock separated into bottom-screen products, as well as top-screen product moisture content. These values are computed with due account for averaged product separation grain-size composition balances shown in [7-13]. Equipment loads and operation parameters met the certificate data. In the process of dry preliminary screening, the nearmesh separation grain size made 50,25, 13 and $6 \mathrm{~mm}$, for wet screening $13 \mathrm{~mm}$, for deslurring $-0.5 ; 1.0$ and $2.0 \mathrm{~mm}$.

\section{Results and discussion}

Total 544 balances of the grain-size composition of products split with screens and aqua screens operated at 102 coal preparation plants were considered.

Size grades separated into bottom-screen products shall be computed by the formula:

$$
\varepsilon_{1, i}=\frac{\gamma_{1 . e x t . i}}{\gamma_{\text {ext. } i}}
$$

where $\gamma_{1 . e x t . i}$ - output (relative to the feedstock) $i$ of the size grade, transferred to the bottom-screen products, $\%$; $\gamma_{\text {ext. } i}$ - output $i$ of feedstock size grade, $\%$.

The ash-content of size grades transferred to the bottom-screen or overflow products $A_{1 . i}^{d}$, is equal to the ash-content of appropriate feedstock size grades $A_{\text {ext.i }}^{d}$, i.e.:

$$
A_{1 . i}^{d}=A_{\text {ext.i }}^{d}, \%
$$

Moisture content reduction factors for the top-screen product $\varepsilon_{\text {top.w }}$ in the process of dry screening:

$$
\varepsilon_{\text {top.w }}=\frac{W_{\text {top }}}{W_{\text {feed }}},
$$

where $W_{\text {top }}, W_{\text {feed }}$ - moisture of appropriate top-screen and feedstock products, $\%$.

Table 1 shows parameters of size grading into bottom-screen products in the process of preliminary dry screening. Table 1 shows relations of coefficients of near-mesh 50-, 25-, 13- and 6-mm size grading into bottom-screen products, thus, as far as the screen operating surface hole size increases, these coefficients get higher. In addition, as far as the near-mesh size is reduced, the bottom-screen product output is drastically decreased. For such size grading the difference between outputs of bottom-screen adjacent screens makes in average $10 \%$ (absolute). In this and the following tables $O_{f . r}$ is a feedstock-relative output, $O_{p . r}$ is a product-relative output, $\varepsilon_{1, c}$ is a calculated value of size grades separated into bottom-screen products.

Table 2 shows computation of moisture content reduction factor for top-screen product $\varepsilon_{\text {top.w }}$ by the formula (3). The provided data show that as far as near-mesh size grading is decreased, top-screen product moisture content reduces as compared with the feedstock moisture content due to separation of the wettest size grades.

Performed studies allow for the conclusion that in the process of preliminary dry screening, 2-time increase or decrease of size grading results in absolute $10 \%$ reduction or 
rise of bottom-screen product output, as appropriate.

Table 1. Computation of parameters of feedstock size grading into the bottom-screen product $\varepsilon_{1}$ in the process of preliminary dry coal screening.

\begin{tabular}{|c|c|c|c|c|c|c|c|c|c|c|}
\hline \multirow{2}{*}{ 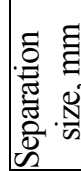 } & \multirow[b]{2}{*}{ Products } & \multirow[b]{2}{*}{ Parameters } & \multicolumn{8}{|c|}{ Grade, mm } \\
\hline & & & $\stackrel{\circ}{f}$ & $\begin{array}{l}\stackrel{0}{1} \\
\stackrel{1}{1}\end{array}$ & $\stackrel{2}{n}$ & $\frac{m}{6}$ & $\begin{array}{l}0 \\
m\end{array}$ & $\stackrel{?}{1}$ & ㄱ & 跑 \\
\hline \multirow{6}{*}{50} & Feedstock & $O_{f, r}, \%$ & 13.8 & 12.5 & 19.5 & 229 & 8.2 & 12.8 & 10.3 & 100.0 \\
\hline & $\begin{array}{c}\text { Top- } \\
\text { screen }\end{array}$ & $\begin{array}{l}\text { Product-relative } \\
\text { output, } \%\end{array}$ & 32.0 & 23.2 & 22.2 & 16.5 & 2.8 & 2.5 & 0.8 & 100.0 \\
\hline & \multirow{4}{*}{$\begin{array}{l}\text { Bottom- } \\
\text { screen }\end{array}$} & $O_{p . r}, \%$ & 2.3 & 5.7 & 17.8 & 27.0 & 11.6 & 19.3 & 16.3 & 100.0 \\
\hline & & $O_{f, r}, \%$ & 1.4 & 3.5 & 10.9 & 16.5 & 7.1 & 11.8 & 10.0 & 61.2 \\
\hline & & $\varepsilon_{1, c}$, unit fraction & 0.10 & 0.28 & 0.56 & 0.72 & 0.87 & 0.92 & 0.97 & \\
\hline & & $\varepsilon_{1}$, unit fraction & 0.10 & 0.30 & 0.55 & 0.70 & 0.85 & 0.90 & 0.95 & \\
\hline \multirow{6}{*}{25} & Feedstock & $O_{f, r}, \%$ & 13.6 & 16.1 & 20.8 & 23.3 & 7.6 & 11.5 & \begin{tabular}{|l|}
7.1 \\
\end{tabular} & 100.0 \\
\hline & Top-screen & $O_{p . r}, \%$ & 21.9 & 25.1 & 24.5 & 20.6 & \begin{tabular}{|l|}
3.7 \\
\end{tabular} & 3.2 & 1.0 & 100.0 \\
\hline & \multirow{4}{*}{$\begin{array}{c}\text { Bottom- } \\
\text { screen }\end{array}$} & $O_{p . r}, \%$ & - & 1.3 & 14.8 & 27.7 & 14.0 & 25.1 & 17.1 & 100.0 \\
\hline & & $O_{f . r}, \%$ & - & 0.5 & 5.6 & 10.5 & 5.3 & 9.5 & 6.5 & 37.9 \\
\hline & & $\varepsilon_{1, c}$, unit fraction & 0 & 0.03 & 0.27 & 0.45 & 0.70 & 0.83 & 0.91 & \\
\hline & & $\varepsilon_{1}$, unit fraction & 0 & 0.05 & 0.25 & 0.45 & 0.70 & 0.85 & 0.90 & \\
\hline \multirow{6}{*}{13} & Feedstock & $O_{f, r}, \%$ & 7.0 & 10.1 & 20.0 & 18.7 & 14.7 & 15.1 & 14.4 & 100.0 \\
\hline & Top-screen & $O_{p . r} \%$ & 9.9 & 14.3 & 27.5 & 21.2 & 12.5 & 9.5 & 5.1 & 100.0 \\
\hline & \multirow{4}{*}{$\begin{array}{l}\text { Bottom- } \\
\text { screen }\end{array}$} & $O_{p . r}, \%$ & - & - & 2.0 & 12.6 & 20.1 & 28.6 & 36.7 & 100.0 \\
\hline & & $O_{f, r}, \%$ & - & - & 0.6 & 3.7 & 5.9 & 8.4 & 10.8 & 29.4 \\
\hline & & $\varepsilon_{1, c}$, unit fraction & 0 & 0 & 0.03 & 0.20 & 0.40 & 0.56 & 0.75 & \\
\hline & & $\varepsilon_{1}$, unit fraction & 0 & 0 & 0.05 & 0.20 & 0.40 & 0.55 & 0.75 & \\
\hline \multirow{6}{*}{6} & Feedstock & $O_{f . r}, \%$ & 10.8 & 7.9 & 15.5 & 14.9 & 19.1 & 18.8 & 13.0 & 100.0 \\
\hline & Top-screen & $O_{p . r}, \%$ & 12.9 & 9.5 & 18.6 & 16.9 & 18.3 & 14.6 & 9.2 & 100.0 \\
\hline & \multirow{4}{*}{$\begin{array}{l}\text { Bottom- } \\
\text { screen }\end{array}$} & $O_{p . r}, \%$ & - & - & - & 4.8 & 23.0 & 40.0 & 32.2 & 100.0 \\
\hline & & $O_{f . r}, \%$ & - & - & - & 0.8 & 3.8 & 6.6 & 5.3 & 16.5 \\
\hline & & $\varepsilon_{1, c}$, unit fraction & 0 & 0 & 0 & 0.05 & 0.20 & 0.35 & 0.41 & \\
\hline & & $\varepsilon_{1}$, unit fraction & 0 & 0 & 0 & 0.05 & 0.20 & 0.35 & 0.40 & \\
\hline
\end{tabular}

Table 2. Computation of moisture content reduction factors for top-screen product $K_{w}$ in the process of preliminary dry coal screening.

\begin{tabular}{|c|c|c|c|c|c|}
\hline \multirow{2}{*}{ Products } & \multirow{2}{*}{ Parameters } & \multicolumn{4}{|c|}{ Size grading, mm } \\
\cline { 3 - 6 } & $W_{u}, \%$ & 50 & 25 & 13 & 6 \\
\hline Feedstock & $W_{h}, \%$ & 5.1 & 6.2 & 7.2 & 6.4 \\
\hline \multirow{2}{*}{ Top-screen } & \begin{tabular}{c}
$\varepsilon_{\text {top.w }}, \begin{array}{c}\text { fraction } \\
\text { unit }\end{array}$ \\
\cline { 3 - 6 }
\end{tabular} & 0.4 & 0.92 & 0.90 & 0.85 \\
\hline
\end{tabular}

Table 3 shows parameters of size grading into the bottom-screen product in the process of preliminary wet screening.

Table 4 shows computation of coefficients of size grading into the bottom-screen product in the process fine machine grade deslurring.

Based upon Table 4, one may conclude that as far as deslurring size is increased, the top-screen product moisture content is decreased. 
Table 3. Computation of parameters of size grading into bottom-screen product in the process of preliminary wet run-of-mine coal screening.

\begin{tabular}{|c|c|c|c|c|c|c|c|c|c|c|}
\hline \multirow[b]{2}{*}{ Products } & \multirow[b]{2}{*}{ Parameters } & \multicolumn{8}{|c|}{ Size grade, $\mathrm{mm}$} & \multirow{2}{*}{ 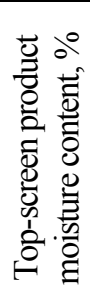 } \\
\hline & & in & $\begin{array}{l}\stackrel{n}{n} \\
\stackrel{n}{n}\end{array}$ & $\begin{array}{l}\text { â } \\
\text { }\end{array}$ & $\frac{m}{b}$ & $\begin{array}{l}0 \\
1 \\
m\end{array}$ & $\stackrel{?}{\perp}$ & $\bar{b}$ & 氶 & \\
\hline \multicolumn{11}{|c|}{ Vibrating screens } \\
\hline \begin{tabular}{|l|} 
Feedstock \\
\end{tabular} & $O_{f . r}, \%$ & 6.4 & 14.4 & 13.4 & 14.9 & 11.7 & 13.5 & 25.7 & 100.0 & \multirow{6}{*}{12.4} \\
\hline Top-screen & $O_{p . r}, \%$ & 18.4 & 38.6 & 25.1 & 8.6 & 3.8 & 2.3 & 3.2 & 100.0 & \\
\hline \multirow{4}{*}{$\begin{array}{c}\text { Bottom- } \\
\text { screen }\end{array}$} & $O_{p, r}, \%$ & 0 & 1.5 & 7.2 & 18.2 & 15.9 & 19.5 & 37.7 & 100.0 & \\
\hline & $O_{f . r}, \%$ & 0 & 1.0 & 4.7 & 11.9 & 10.4 & 12.7 & 24.6 & 65.3 & \\
\hline & $\varepsilon_{1, c}$, unit fraction & 0 & 0.07 & 0.35 & 0.80 & 0.89 & 0.94 & 0.96 & & \\
\hline & $\varepsilon_{1}$, unit fraction & 0 & 0.05 & 0.35 & 0.80 & 0.90 & 0.94 & 0.96 & & \\
\hline \multicolumn{11}{|c|}{ Vibrating screen machines } \\
\hline \begin{tabular}{|l|} 
Feedstock \\
\end{tabular} & $O_{f . r}, \%$ & 10.1 & 14.0 & 14.8 & 20.1 & 12.1 & 12.9 & 16.0 & 100,0 & \multirow{6}{*}{9.8} \\
\hline Top-screen & $O_{p, r}, \%$ & 23.3 & 32.3 & 31.4 & 8.6 & 2.3 & 1.4 & 0.7 & 100.0 & \\
\hline \multirow{4}{*}{$\begin{array}{c}\text { Bottom- } \\
\text { screen }\end{array}$} & $O_{p . r}, \%$ & 0 & 0 & 2.1 & 28.9 & 19.6 & 21.7 & 27.7 & 100.0 & \\
\hline & $O_{f . r}, \%$ & 0 & 0 & 1.2 & 16.4 & 11.1 & 12.3 & 15.7 & 56.7 & \\
\hline & $\varepsilon_{1, c}$, unit fraction & 0 & 0 & 0.08 & 0.81 & 0.92 & 0.95 & 0.98 & & \\
\hline & $\varepsilon_{1}$, unit fraction & 0 & 0 & 0.10 & 0.80 & 0.90 & 0.95 & 0.98 & & \\
\hline
\end{tabular}

Based upon the data of Tables 1 - 4, the procedure for computation of qualitative and quantitative parameters of run-of-mine coal screening with screens and coal slurry ranging with aqua screens includes the following.

$i$-size grade output into bottom-screen product:

$$
\gamma_{1 i}=\varepsilon_{1 i} \cdot \gamma_{\text {feed.i } i}, \%
$$

where $\varepsilon_{1 i}$ - coefficient of $i$-size grading into bottom-screen or overflow product, unit friction, shall be assumed in line with Tables $1-4 ; \gamma_{\text {feed. } i}-i$-size grade output in the feedstock product.

$i$-size grade output into top-screen or over-split product $\gamma_{2 i}$ :

$$
\gamma_{2 i}=\left(1-\varepsilon_{1 i}\right) \cdot \gamma_{f e e d . i}, \%
$$

The ash-content of $i$-size grades in the bottom-screen products $A_{1 i}^{d}$ and top-screen products $A_{2 i}^{d}$ shall be equal to the ash-content of $i$-size grade in the feedstock product $A_{\text {feed.i }}^{d}$, i.e.:

$$
A_{1 i}^{d}=A_{2 i}^{d}=A_{\text {feed. } i}^{d}, \% .
$$

Bottom-screen product output $\gamma_{1}$ :

$$
\gamma_{1}=\sum_{i=1}^{n} \gamma_{1 i}
$$


Table 4. Computation of parameters of size grading into the bottom-screen product in the process of fine machine grade deslurring.

\begin{tabular}{|c|c|c|c|c|c|c|c|c|c|c|c|}
\hline \multirow{2}{*}{ 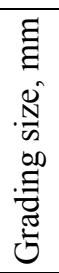 } & \multirow[b]{2}{*}{ Products } & \multirow[b]{2}{*}{$\begin{array}{l}\text { Para- } \\
\text { meters }\end{array}$} & \multicolumn{8}{|c|}{ Grade, mm } & \multirow{2}{*}{ 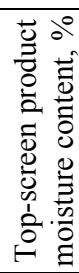 } \\
\hline & & & $\begin{array}{l}a ̂ \\
\\
\end{array}$ & $\frac{m}{b}$ & $\begin{array}{l}0 \\
1 \\
1\end{array}$ & $\stackrel{?}{1}$ & $\stackrel{1}{n}$ & 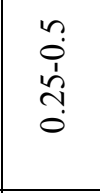 & $\begin{array}{l}\text { ก̣ } \\
\text { } \\
0\end{array}$ & 要 & \\
\hline \multirow{6}{*}{0.5} & Feedstock & $O_{f, r}, \%$ & 3.3 & 20.3 & 17.9 & 17.5 & 13.1 & 11.4 & 16.5 & 100.0 & \multirow{6}{*}{40.0} \\
\hline & $\begin{array}{c}\text { Top- } \\
\text { screen }\end{array}$ & $O_{p . r}, \%$ & 4.2 & 26.0 & 22.9 & 22.3 & 13.5 & 6.8 & 4.3 & 100.0 & \\
\hline & \multirow{4}{*}{$\begin{array}{l}\text { Bottom- } \\
\text { screen }\end{array}$} & $O_{p . r}, \%$ & 0 & 0 & 0 & 0.4 & 11.8 & 27.8 & 60.0 & 100.0 & \\
\hline & & $O_{f . r}, \%$ & 0 & 0 & 0 & 0.09 & 2.57 & 6.06 & 13.08 & 21.8 & \\
\hline & & $\begin{array}{l}\varepsilon_{1, c}, \text { unit } \\
\text { fraction }\end{array}$ & 0 & 0 & 0 & 0.005 & 0.196 & 0.532 & 0.794 & & \\
\hline & & $\begin{array}{l}\varepsilon_{1}, \text { unit } \\
\text { fraction }\end{array}$ & 0 & 0 & 0 & 0.01 & 0.20 & 0.55 & 0.80 & & \\
\hline \multirow{6}{*}{1.0} & Feedstock & $O_{f . r}, \%$ & 7.8 & 17.4 & 18.1 & 22.0 & 11.1 & 11.1 & 12.5 & 100.0 & \multirow{6}{*}{35.0} \\
\hline & Top-screen & $O_{p . r}, \%$ & 10.7 & 23.9 & 24.6 & 25.4 & 8.3 & 4.6 & 2.5 & 100.0 & \\
\hline & \multirow{4}{*}{$\begin{array}{c}\text { Bottom- } \\
\text { screen }\end{array}$} & $O_{p . r}, \%$ & 0 & 0 & 0.6 & 12.9 & 18.7 & 28.6 & 39.2 & 100.0 & \\
\hline & & $O_{f . r}, \%$ & 0 & 0 & 0.16 & 3.52 & 5.09 & 7.77 & 10.66 & 27.2 & \\
\hline & & $\begin{array}{l}\varepsilon_{1, c}, \text { unit } \\
\text { fraction }\end{array}$ & 0 & 0 & 0.009 & 0.160 & 0.458 & 0.700 & 0.853 & & \\
\hline & & $\begin{array}{l}\varepsilon_{1}, \text { unit } \\
\text { fraction }\end{array}$ & 0 & 0 & 0.01 & 0.15 & 0.45 & 0.70 & 0.85 & & \\
\hline \multirow{6}{*}{2.0} & Feedstock & $O_{f . r}, \%$ & 6.7 & 18.6 & 13.4 & 23.4 & 12.1 & 10.8 & 15.0 & 100.0 & \multirow{6}{*}{25.0} \\
\hline & Top-screen & $O_{p . r}, \%$ & 10.7 & 29.6 & 19.1 & 28.0 & 6.8 & 3.5 & 2.3 & 100.0 & \\
\hline & \multirow{4}{*}{$\begin{array}{c}\text { Bottom- } \\
\text { screen }\end{array}$} & $O_{p . r}, \%$ & 0 & 0.4 & 3.9 & 15.6 & 21.0 & 23.0 & 36.3 & 100.0 & \\
\hline & & $O_{f . r}, \%$ & 0 & 0.15 & 1.46 & 5.85 & 7.87 & 8.63 & 13.54 & 37.5 & \\
\hline & & $\begin{array}{l}\varepsilon_{1, c}, \text { unit } \\
\text { fraction }\end{array}$ & 0 & 0.008 & 0.109 & 0.250 & 0.651 & 0.799 & 0.903 & & \\
\hline & & $\begin{array}{l}\varepsilon_{1}, \text { unit } \\
\text { fraction }\end{array}$ & 0 & 0.01 & 0.10 & 0.25 & 0.65 & 0.80 & 0.90 & & \\
\hline
\end{tabular}

The ash content of the bottom-screen product $A_{1}^{d}$ :

$$
A_{1}^{d}=\left(\sum_{i=1}^{n} \gamma_{1 i} \cdot A_{1 i}^{d}\right) / \gamma_{1}, \%
$$


Top-screen product output $\gamma_{2}$ :

$$
\gamma_{2}=\sum_{i=1}^{n} \gamma_{2 i}, \%
$$

The ash content of the top-screen product $A_{2}^{d}$ :

$$
A_{2}^{d}=\left(\sum_{i=1}^{n} \gamma_{2 i} \cdot A_{2 i}^{d}\right) / \gamma_{2}, \%
$$

Upon definition of the grain size compositions of the split products and using fraction compositions of run-of-mine coal size grades, fraction compositions of machine grades and their preparability classes may be computed, hence, the rates of product separation wasting of subsequent preparation operations.

\section{Conclusions}

1. Parameters of run-of-mine coal size grading into the bottom-screen product in the process of preliminary dry and wet run-of-mine coal screening and fine machine grade deslurring, as well as into the aqua screen overflow product in the process of coal slurry ranging have been computed.

2 . The averaged moisture content of the top-screen products of the screens under study has been evaluated.

3. These dependences may be used for computation of qualitative and quantitative parameters of run-of-mine coal machine grade preparation operations and utilizing fraction compositions of run-of-mine coal, fraction compositions of machine grades and their preparability classes may be computed, hence, the rates of product separation wasting of subsequent preparation operations.

\section{References}

1. Bulat, A.F., Skypochka, S.I., Palamarchuk, T.A. (2010). Metanogeneracia v ugolnyh plastah [Methane generation in coal seams]. Dnipropetrovsk: Lira

2. Bulat, A.F., Skypochka, S.I., Palamarchuk, T.A. (2009). Metodicheskiye rekomendatsiyi po geofizicheskomu kontrolu i diagnostike geomehanicheskogo sostoyaniya podzemnyh goetehnicheskyh sistem ugolnyh shaht [Methodical recommendations about geophysical control and diagnostics of geomechanical state of underground geotechnical system of coal mines], Donetsk-Dnipropetrovsk: VIK

3. T. Xu, C.A. Tang, T.H. Yang, Numerical investigation of coal and gas outbursts in underground collieries, International Journal of Rock Mechanics \& Mining Sciences, 4, 905-919 (2006)

4. Vynogradov, V.V. (1989). Geomehanika upravleniya sostoyaniyem massiva vblizi gornykh vyrabotok [Geomechanic of control by the state of array near the rock making]. Kyiv: Naukova dumka

5. Nikolaevskiy, V.N. (1996). Geomehanika $i$ fluidodinamika [Geomechanics and fluidodynamics]. Moskva: Nedra

6. Bulat, A.F., Skipochka, S.I., Pilipenko, Yu.N., Dyakun, R.A. (2016). Scientific and methodical basis for spectral-acoustic state control of fluid-saturated coal massif, Geotechnical Mechanics, 114, 4-16 
7. Kurlenya, M.V., Serdukov, A.S., Serdukov, S.V., Cheverda, V.A. (2010). Localization of methane accumulation centers in coal seam by seismic method, FTPRPI, 6, 37-47

8. Antsiferov, V.A. (2003). Teoriya i praktika shahtnoy seismorazvedky [Theory and practice of mine seismic exploration]. Donetsk: Alan 